\title{
Effects of various factors on Doppler flow ultrasonic radial and coccygeal artery systolic blood pressure measurements in privately-owned, conscious dogs
}

\author{
Allison P Mooney ${ }^{1}$ ， Dianne I Mawby ${ }^{1}$, Joshua M Price $^{2}$, Jacqueline C Whittemore $^{\text {Corresp. }}{ }^{1}$ \\ 1 Department of Small Animal Clinical Sciences, University of Tennessee - Knoxville, Knoxville, Tennessee, United States \\ Corresponding Author: Jacqueline C Whittemore \\ Email address: jwhittemore@utk.edu
}

Objective. The purpose of this study was to assess the effects of age, body condition score (BCS) and muscle condition score (MCS) on indirect radial and coccygeal Doppler systolic arterial blood pressure (SAP) measurements in dogs.

Methods. Sixty-two privately-owned dogs were enrolled between June and July 2016. The BCS and MCS were determined by 2 investigators. Blood pressure was measured per published guidelines and using headphones, and the order of measurement site was randomized. Dogs were positioned in right lateral recumbency for radial measurements and sternal recumbency or standing for coccygeal measurements. Associations between SAP and other variables were assessed by correlation coefficients and analysis of covariance.

Results. Radial and coccygeal SAP measurements were moderately correlated $(r=0.45, P<0.01)$. Radial SAP measurements were higher than coccygeal SAP measurements (mean difference $9 \mathrm{mmHg}, P<0.01$ ), but discordance occurred in both directions. No difference was observed between the first measurement taken, the average of measurements $2-6$, or the average of all 6 measurements for either the radial (128, 129 , and $129 \mathrm{mmHg} ; P=0.36)$ or coccygeal $(121,122$, and $122 \mathrm{mmHg} ; P=0.82)$ site. Associations were not found between SAP measurements for either site and age, weight, BCS, MCS, anxiety score, or cuff size. Heart rate decreased significantly from the start of acclimation to the end of the first data collection series regardless of site $(P<0.01)$.

Conclusions and Clinical Relevance. Initial measurement site can be based on patient and operator preference given lack of associations with patient variables, but the same site should be used for serial SAP measurements given discordant results between sites. 
1 Effects of various factors on Doppler flow ultrasonic radial and coccygeal artery systolic

2 blood pressure measurements in privately-owned, conscious dogs

3 Allison P. Mooney ${ }^{1}$; Dianne I. Mawby, DVM, MVS, DACVIM; ${ }^{1}$ Joshua M. Price, $M S ;^{2}$ Jacqueline $^{2}$

4 C. Whittemore, DVM, PhD, DACVIM-1

5

$6 \quad{ }^{1}$ Department of Small Animal Clinical Sciences, University of Tennessee College of

7 Veterinary Medicine, University, Knoxville, TN, United States

$8{ }^{2}$ Office of Information Technology, University of Tennessee College of Veterinary Medicine,

9 University, Knoxville, TN, United States

10

11 Corresponding Author:

12 Jacqueline Whittemore ${ }^{1}$

13 Department of Small Animal Clinical Sciences, College of Veterinary Medicine, University of

14 Tennessee, 2407 River Drive, Knoxville, TN 37996, United States

15 Email address: jwhittemore@utk.edu 


\section{Abstract}

19

20 Objective. The purpose of this study was to assess the effects of age, body condition score

21 (BCS) and muscle condition score (MCS) on indirect radial and coccygeal Doppler systolic

22 arterial blood pressure (SAP) measurements in dogs.

23

24 Methods. Sixty-two privately-owned dogs were enrolled between June and July 2016.

25 The BCS and MCS were determined by 2 investigators. Blood pressure was measured per

26 published guidelines and using headphones, and the order of measurement site was randomized.

27 Dogs were positioned in right lateral recumbency for radial measurements and sternal

28 recumbency or standing for coccygeal measurements. Associations between SAP and other

29 variables were assessed by correlation coefficients and analysis of covariance.

31 Results. Radial and coccygeal SAP measurements were moderately correlated $(r=0.45, P<0.01)$.

32 Radial SAP measurements were higher than coccygeal SAP measurements (mean difference $339 \mathrm{mmHg}, P<0.01$ ), but discordance occurred in both directions. No difference was observed 34 between the first measurement taken, the average of measurements 2-6, or the average of all 6 35 measurements for either the radial $(128,129$, and $129 \mathrm{mmHg} ; P=0.36)$ or coccygeal $(121,122$, and $36122 \mathrm{mmHg} ; P=0.82$ ) site. Associations were not found between SAP measurements for either site 37 and age, weight, BCS, MCS, anxiety score, or cuff size. Heart rate decreased significantly from 38 the start of acclimation to the end of the first data collection series regardless of site $(P<0.01)$. 
40 Conclusions and Clinical Relevance. Initial measurement site can be based on patient and

41 operator preference given lack of associations with patient variables, but the same site should be

42 used for serial SAP measurements given discordant results between sites.

43 
45

46

47

48

49

50

51

52

53

54

55

56

57

58

59

60

61

62

63

64

65

66

67

\section{Introduction}

Due to the lack of overt clinical signs directly attributable to systemic hypertension, it is considered one of the most under-diagnosed systemic illnesses in companion animals (Acierno \& Labato, 2004; Brown et al., 2007). Prolonged systemic hypertension is associated with damage to the kidneys, eyes, brain, and heart (Brown \& Henik, 1998; Brown et al., 2007; Hsiang, Lien \& Huang, 2008; Carr \& Egner, 2009). In dogs, systemic hypertension can occur secondary to diseases including kidney disease, hyperadrenocorticism, and diabetes mellitus (Bodey \&Michell, 1996; Brown \& Henik, 1998; Brown et al., 2007; Hsiang, Lien \& Huang, 2008; Carr \& Egner, 2009). Due the impact of hypertension on long-term outcome, routine surveillance for hypertension is recommended for dogs displaying signs consistent with end organ damage or that have been diagnosed with diseases or conditions associated with secondary hypertension (Acierno \& Labato, 2004; Henik, Dolson \& Wenholz, 2005; Brown et al., 2007). While there is some debate regarding the relationship between advancing age and hypertension, conditions that can cause secondary hypertension are more often observed in geriatric pets, so this population should be monitored for the development of those diseases (Brown et al., 2007).

Hypotension requiring vasopressor therapy is associated with a 3.4-fold increase in the risk of death in dogs with sepsis (Kenney et al., 2010). Silverstein, et al. further demonstrated that an increase in systolic Doppler blood pressure of $\geq 20 \mathrm{mmHg}$ increased the chance of survival to discharge in hypotensive cats (Silverstein et al., 2008) and that dogs that had normalization of hypotension within the first hour of fluid resuscitation were more likely to survive to discharge (Silverstein et al., 2012). Thus, accurate determination of indirect blood pressure measurement is key to identify patients with both hyper- and hypotension in order to optimize outcome. 
Although direct arterial measurement of blood pressure remains the gold standard, the pain and specialized equipment associated with this method limits its use in a clinical setting (Sawyer at al., 1991; Gains et al., 1995; Bodey et al., 1996). Indirect methods of measuring blood pressure are less invasive and technically demanding and are, therefore, more practical for routine monitoring of blood pressure in conscious dogs. Currently, the two most frequently used modalities of indirect blood pressure measurement are ultrasonic Doppler flow monitors and automated oscillometric devices. The Doppler flow method has been shown to be more efficient and generate more precise measurements of systolic arterial blood pressure (SAP) in conscious dogs in a clinical setting (Hsiang, Lien \& Huang, 2008; Wernick et al., 2012). Furthermore, oscillometry can underestimate increased SAP and overestimate decreased SAP, resulting in failure to diagnose hyper- and hypotension, respectively (Sawyer et al., 1991; Gains et al., 1995; Bodey et al., 1996; Wernick et al., 2012; Vachon, Belinger \& Burns, 2014). In addition to the variability that exists between the two methods described above, both the location of cuff placement, as well as the body position of the animal, can significantly affect the accuracy of indirect blood pressure measurements (Bodey et al., 1994; Bodey et al., 1996; Rondeau, Mackalonis \& Hess, 2013; Scansen et al., 2014). Selection of an appropriately-sized blood pressure cuff also impacts the accuracy of indirect blood pressure measurements, with cuff-size inversely correlated with blood pressure measurement results (Valtonen \& Eriksson, 1970; Bodey et al., 1994; Sparkes et al., 1999). Excitement or anxiety experienced during the measurement process, a phenomenon known as white-coat hypertension, can also result in erroneous measurement results (Belew, Barlett \& Brown, 1999; Brown et al., 2007; Bragg et al., 2015). Finally, patient-specific factors can affect the accuracy of indirect blood pressure measurements. Although no association has been identified between body condition score (BCS) 
91 or weight and indirect blood pressure measurement results in either cats (Sparkes et al., 1999) or

92 dogs (Remillard, Ross \& Eddy, 1991; Bosiack, et al., 2010), Whittemore et al. (JAVMA, in

93 press) recently found that muscle mass is inversely associated with SAP measurements taken

94 using the radial, but not the coccygeal, artery in cats. Prior studies assessing the effects of weight

95 and BCS on SAP measurements did not separately assess muscle condition score (MCS). To the

96 authors' knowledge, the separate effects of BCS and MCS on correlations between indirect radial

97 and coccygeal SAP measurements in dogs are unknown.

98 The purpose of this study was to assess the impacts of BCS and MCS on correlations

99 between indirect radial and coccygeal SAP measurements taken using the Doppler method in

100 privately-owned dogs. Secondary objectives of the study were to assess for associations between

101 SAP measurement results and anxiety score, cuff size, and heart rate and to compare time

102 required for collection of a complete series of readings at each site.

103

104 Materials \& methods

105 Study population - This study was conducted at the University of Tennessee's

106 Veterinary Medical Center and was approved by the Institutional Animal Care and Use

107 Committee of the University of Tennessee, Knoxville (protocol number 2426).

108 Between June and July 2016, privately-owned dogs were enrolled in the study based on

109 initial BCS determination assigned by 1 investigator (APM) using a 5-point scale (Baldwin et al.,

110 2010). An enrollment goal of 20 dogs each per whole integer BCS was established for a total

111 target sample size of 100 dogs. After 20 dogs had been enrolled within a whole integer score, no

112 additional dogs of the same BCS were enrolled. 
114 belonging to faculty, staff, and students were recruited. Informed consent was obtained for each

115 dog prior to enrollment. Prior to data collection, owners were asked to provide information

116 regarding any known medical conditions and/or current medications. Dogs that did not have a

117 tail, had been anesthetized within the previous $12 \mathrm{hrs}$, or became fractious or intolerant of

118 manipulation were excluded from the study. Participants diagnosed with local or systemic

119 diseases were not excluded, nor were those dogs receiving medications, consistent with previous

120 studies (King et al., 2001; Hsiang, Lien \& Huang, 2008; Bosiack et al., 2010; Wernick, 2012).

121 Immediately upon entry into the exam room, both the weight and initial heart rate were recorded.

122 Determination of BCS and MCS - All investigators that completed BCS and MCS

123 scoring received training in BCS and MCS assignment from a board-certified veterinary

124 nutritionist prior to the start of data collection. Investigators independently assigned a BCS and

125 MCS to each participant using a 5-point scale and a 4-point scale, respectively (Baldwin et al.,

126 2010; Michel, 2011). The initial BCS used for sample stratification was assigned to each dog by

127 one investigator (APM). Body condition scores and MCS from two additional investigators

128 (DIM, JCW) were used for statistical analyses. These investigators were blinded to BCS and

129 MCS scores for the other investigators, as well as all SAP measurement results, until completion

130 of sample collection for the study.

131 Randomization - Using a randomized number generator ${ }^{\mathrm{a}}$, a randomization table was

132 generated to determine the initial site of SAP measurement (radial versus coccygeal artery) for

133 the target number of participants for each BCS category. Based on the initial BCS assigned,

134 dogs were allocated into measurement groups using the next available assignment in the

135 randomization table. If a participant was excluded from the study after assignment in the 
136 randomization table, its name was crossed out once on the table and that randomization

137 assignment was recycled for the next participant.

138 Blood pressure measurement - Blood pressure measurements were obtained following

139 the guidelines provided in the American College of Veterinary Internal Medicine Consensus

140 Statement (Brown et al., 2007). Briefly, both the dog and owner (if possible) were brought into a

141 quiet exam room for data collection. If the owner was unable to be present for both pressure

142 series, then either the investigator collected all measurements without assistance or a veterinary

143 assistant assisted with the entire data collection series. The circumference of both the left mid-

144 antebrachium and the base of the tail were measured using a soft measuring tape, and cuff size

145 was selected to be approximately $40 \%$ of the circumference of each appendage. The location

146 where the Doppler crystal was to be placed for the first pressure series was shaved - on the

147 palmar surface of the left front foot between the carpal and metacarpal pads or the ventral surface

148 of the tail just distal to site of cuff placement. The dog was then allowed to acclimate for 10

149 minutes to the environment and measurement personnel. Heart rates at the beginning and end of

150 acclimation were recorded, as well as 'start' and 'stop' times for the acclimation period.

151 After acclimation was complete, the blood pressure cuff was applied to the first

152 measurement site as per group assignment, either just proximal to the carpus on the left mid-

153 antebrachium or just distal to the base of the tail. For radial SAP measurements, dogs were

154 placed in right lateral recumbency with the left forelimb positioned so that the cuff was at the

155 same level as the heart. If the left antebrachium was unavailable for measurements (due to

156 injury, IV catheter placement, etc.), the animal was placed in left lateral recumbency and the

157 right antebrachium was used. For coccygeal SAP measurements, the dog was allowed to either

158 stand or lay in sternal recumbency. Ultrasonic coupling gel was applied to the concave side of a 
159 flat infant Doppler probe, which was then positioned perpendicular over the artery. Using the

160 same sphygmomanometer ${ }^{b}$ and Doppler unit with probe ${ }^{c}$ for every SAP measurement, the cuff

161 was inflated to approximately $20 \mathrm{mmHg}$ above the point at which blood flow was no longer

162 audible. Air was slowly and completely released from the cuff, and the pressure at which flow

163 was first heard was recorded as the SAP. The process was repeated until 6 consistent readings

164 were obtained. Heart rates at the beginning and end of each pressure series were recorded using

165 Doppler ultrasonic pulse detection. Headphones were worn by the investigator for the entire

166 duration that the Doppler was turned on to limit the potential of noise from the machine to

167 contribute to 'white coat' effects. An anxiety score (ranging from 0-3) was assigned to the

168 participant after completion of data collection (Scansen et al., 2014), with a calm dog requiring

169 no restraint being assigned a 0 and a highly anxious dog requiring active restraint assigned a 3.

170 As noted above, dogs that were refractory to SAP measurement at either site were excluded from

171 the study. After completion of data collection at the first site, instrumentation was removed and

172 the alternate site was clipped. Then the measurement procedure was repeated for the alternate

173 site starting with the 10-minute acclimation period.

174 Data entry - After data collection was completed on a subject, as described above, the

175 data collection sheet was turned in to a person unrelated to study. That person was responsible

176 for entry of all results into the study database, which was not available to any of the investigators

177 until after completion of data collection for all subjects in the study.

178 Statistical analyses - Descriptive statistics were generated for each parameter.

179 Continuous measures were analyzed for normality using the Shapiro-Wilk test and for the

180 presence of outliers using the box-and-whisker plots. Equality of variances was analyzed with

181 Levene's test for equality of variances. Parameters with normally distributed data were reported 
182 as mean \pm standard deviation, with non-parametrically distributed data reported as median

183 (range). Interclass correlation coefficients (weighted $\kappa$ ) were calculated for inter-rater reliability

184 for BCS and MCS scores assigned by two investigators (DIM, JCW), after which the mean of the

1852 scores was used for all further analyses. Mean systolic blood pressure measurements greater

186 than $150 \mathrm{mmHg}$ were considered consistent with hypertension, based on previous guidelines

187 (Brown et al., 2007). Mean SAP measurements less than $90 \mathrm{mmHg}$ were considered consistent

188 with hypotension (Ateca et al., 2015). Repeated measures analysis of variance (ANOVA) was

189 used to assess for differences in heart rate at the start and conclusion of each measurement series,

190 as well as between the initial SAP reading, the mean of blood pressure measurements 2-6 (BP 2-

191 6), and the mean of all 6 readings for each site. In keeping with ACVIM consensus guidelines

192 (Brown et al., 2007), the mean of BP 2-6 was used for all remaining analyses. Finally, a paired

193 sample student's $t$ test was used to compare time required for completing data collection at each

194 site.

195

Pearson product moment correlation coefficients were calculated to assess correlations

196 between the average of BP 2-6 for the radial and coccygeal sites, as well as between the average

197 of BP 2-6 for each site and possible covariates (age, weight, heart rate, BCS, MCS, and limb or

198 tail circumference as appropriate). Regression analysis was used to determine variance inflation

199 factors to assess for collinearity between possible covariates (age, weight, the mean of starting

200 and final heart rate for each pressure series, BCS, MCS, anxiety score, and cuff size as a percent

201 of appendage circumference).

202 A mixed effects crossover design and corresponding ANCOVA was performed to

203 determine if mean BP 2-6 differed between radial and coccygeal measurement sites and to assess

204 whether the washout period between treatment sites was adequate. Period and Site were included 
205 as fixed effects. Age, weight, average heart rate, BCS, MCS, anxiety score, time required to

206 complete the measurement series, and cuff size as a percentage of appendage circumference were

207 initially included as covariates in the analysis. Dog nested within sequence was included as a

208 random effect. A compound symmetry variance/covariance structure was incorporated into the

209 model to account for constant covariates. Backwards variable selection was performed on the

210 full model to determine which covariates explain significant variability in mean BP 2-6. Factors

211 included in the final model were period, site, and average heart rate. The Shapiro-Wilk test of

212 normality of the residuals was evaluated to ensure the assumptions of the statistical method had

213 been met. Commercial statistical software packages ${ }^{\mathrm{d}, \mathrm{e}, \mathrm{f}}$ were used for all analyses. $P<0.05$ was

214 considered significant.

215

216 Results

217 Seventy dogs were enrolled in the study, of which 8 were excluded due to intolerance of

218 manipulation or SAP measurement at either the radial or coccygeal artery $(n=5)$ or unavailability

219 of one of two investigators (JCW, DIM) to assign a BCS/MCS prior to subject discharge $(\mathrm{n}=3)$.

220 Initial BCS categorization for the 62 dogs that completed the study was BCS 1: 2 dogs, BCS 2: 6

221 dogs, BCS 3: 20 dogs, BCS 4: 20 dogs, and BCS 5: 14 dogs. Age, sex distribution, weight,

222 anxiety score, mean BCS and MCS for measurements assigned by DIM and JCW, and mean

223 radial and coccygeal SAP measurements are presented in Table 1. Mixed breed dogs comprised

224 the majority of the study population $(n=24)$, with 3 or less each of 29 breeds. The complete list

225 of breeds and the number of dogs representative of those breeds are provided in Supplemental

226 Table 1. Of the 62 dogs that completed the study, 33 dogs were healthy and 29 had one or more

227 known disease processes or conditions (see data file for individual diagnoses). The interclass 
228 correlation coefficients for inter-rater reliability for BCS and MCS as assigned by DIM and JCW

229 were $\kappa=0.76(P<0.01)$ and $\kappa=0.57(P<0.01)$, respectively.

As noted above, heart rate was determined at 4 time points throughout each measurement

231 procedure (start of acclimation, end of acclimation, start of SAP measurements, end of SAP

232 measurements). Mean heart rate differed significantly over time $(P<0.01)$ at each site. For the

233 radial site, heart rate values ranged from $104 \mathrm{bpm}$ (range: 68-180) at the beginning of the

234 acclimation period to $93 \mathrm{bpm}$ (range: 54-162) at the end of the procedure. Post-hoc tests

235 indicated that radial heart rate values were significantly different at the beginning of the

236 acclimation period when compared to heart rates at the 3 other time points $(\mathrm{P}<0.01)$. For the

237 coccygeal site, heart rate ranged from $104 \mathrm{bpm}$ (range: 60-180) at the beginning of the

238 acclimation period to $93 \mathrm{bpm}$ (range: 60-162) at the end of the measurement period. Although

239 there was a significant difference in heart rate during the acclimation period $(\mathrm{P}<0.01)$, this

240 difference was not evident across all time points based on post-hoc analysis. Interestingly, there

241 was no significant difference between the first blood pressure taken, the average of BP 2-6, or

242 the average of all 6 measurements for either the radial $(128,129$, and $129 \mathrm{mmHg}$, respectively; $P$

$243=0.36)$ or coccygeal $(121,122$, and $122 \mathrm{mmHg}$, respectively; $P=0.82)$ site. There was also no

244 difference in the time required for collection of the complete SAP measurement series for the

245 two sites ( 5 minutes for the radial site $v s 4.6$ minutes for the coccygeal site; $P=0.30$ ).

246 One dog had a history of severe degenerative joint disease in the lower spine and tail and

247 was markedly distressed by movements near, or manipulation of, the hind end. This dog had

248 marked discordance in SAP readings (radial, $113 \mathrm{mmHg} v s$ coccygeal, $224 \mathrm{mmHg}$ ) and was

249 censored from the analyses of BP 2-6 results because collection of coccygeal measurements

250 would not have been performed in this case given prior knowledge of its orthopedic disease. 
251 Even after censoring results for the previously described dog, radial and coccygeal artery blood

252 pressure measurements were poorly correlated ( $r=0.45, P<0.01$, Figure 1). Passing-Bablok

253 regression and Bland-Altman analyses did not reveal either constant or proportional bias.

254 Discordance in radial and coccygeal artery SAP measurements occurred in both

255 directions across all BCS (Figure 2) and MCS. There were no significant correlations between

256 mean BP 2-6 for either site and any of the assessed possible covariates. Further, variance

257 inflation factors were within the normal range for age, weight, the mean of starting and final

258 heart rates for each SAP measurement series, BCS, MCS, anxiety score, and cuff size as a

259 percent of appendage circumference. Based on lack of evidence of collinearity, all were included

260 in the initial mixed effects crossover design. Age, weight, BCS, MCS, anxiety score, and cuff

261 size as a percent of appendage circumference were not correlated with BP2-6 $(P>0.2)$. Thus, the

262 final crossover model included measurement period, site, and mean heart rate. Of these, there

263 was no significant effect of measurement period $(P>0.2)$ on BP 2-6 results, thereby indicating

264 that the washout period between treatments was sufficient. Radial artery BP 2-6 results

265 significantly differed from coccygeal results (F-value 18.9, $P<0.01$ ). Mean radial BP 2-6 was

266 higher than coccygeal BP 2-6 (mean difference 9mmHg, $P<0.01$ ), but discordance occurred in

267 both directions. A significant positive association was also found between BP 2-6 and mean

268 heart rate (F-value 5.9, $P=0.02)$.

269 Mean radial and coccygeal BP 2-6 were $129 \pm 18.0 \mathrm{mmHg}$ and $120 \pm 18.8 \mathrm{mmHg}$,

270 respectively. Of the 62 dogs, $8(12.9 \%)$ were categorized as hypertensive based on radial SAP

271 measurements, vs $6(9.7 \%)$ dogs based on coccygeal SAP measurements. Two dogs with

272 histories of refractory hypertension $(>190 \mathrm{mmHg}$ ) that were enrolled in the study were 
273 normotensive (mean $140 \mathrm{mmHg}$ ). No dogs were categorized as hypotensive based on radial SAP

274 measurements vs. 2 (3.2\%) dogs based on coccygeal SAP measurements.

275

276

277 Discussion

278

Radial and coccygeal artery blood pressure measurements were poorly correlated in this

279 study. Although discordance between measurements taken at these two sites occurred in both

280 directions, mean radial BP2-6 measurements were significantly higher than those obtained at the

281 coccygeal artery. Consistent with previous reports (Remillard, Ross \& Eddy, 1991; Bosiack et

282 al., 2010), no significant associations were found between BP2-6 at either site and age, BCS, or

283 weight. Furthermore, no association was found between MCS and BP2-6, in contrast to results

284 from a recent study in cats (Whittemore et al., JAVMA, in press). Importantly, this finding

285 indicates that the presence of sarcopenia need not affect site selection for indirect SAP

286 measurements taking using the Doppler flow method in dogs. Thus, individual patient factors

287 can be prioritized when selecting a blood pressure measurement site, the importance of which is

288 underscored by the marked discordance in results in one dog with severe degenerative joint

289 disease in this study.

290 Several previous studies have also identified discordance between indirect and direct

291 blood pressure measurements taken at different sites (Bodey \& Michell, 1996; Rondeau,

292 Mackalonis, \& Hess, 2013; Acierno et al., 2015). Bodey and Michell found oscillometric

293 systolic blood pressure measurements were significantly lower at the coccygeal artery compared

294 to the radial artery in standing dogs (Bodey \& Michell, 1996). In contrast, the opposite was

295 found for oscillometric blood pressure measurements taken in lateral recumbency in the same 
296 study (Bodey \& Michell, 1996). The disagreement between our results and those obtained by

297 Bodey and Michell in laterally recumbent dogs could be attributed to the differences in body

298 position and the wide variety of dog breeds and conformations sampled in our study. Instead of

299 requiring dogs to assume a standard position for tail measurements, participants were allowed to

300 either remain standing or lie in sternal recumbency. It is possible that the difference in the level

301 of the coccygeal artery relative to the heart was greater in larger, deep-chested dogs allowed to

302 remain standing than in smaller dogs of more moderate to shallow-chested conformation. This

303 could have led to a greater discrepancy in results than would have been observed had the dogs all

304 been positioned in sternal recumbency for coccygeal readings. The impact of body position on

305 indirect blood pressure measurement is further highlighted by the results of Rondeau,

306 Mackalonis, and Hess, in which the mean SAP in conscious dogs measured on the forelimb was

307 significantly higher in the sitting position than in lateral recumbency (Rondeau, Mackalonis \&

308 Hess, 2013). Finally, significant differences have been noted in direct SAP measurements

309 collected at different anatomic sites, particularly at the carpus and hindlimb (Acierno et al.,

310 2015), suggesting an anatomic basis for discordance in pressures at different sites. Ultimately,

311 the results of these studies underscore the importance of consistency in both measurement site

312 and body positioning when obtaining indirect blood pressure readings, especially when

313 monitoring for the progression of disease.

314 White-coat hypertension refers to an increase in blood pressure secondary to the stress

315 and anxiety associated with the measurement process (Belew, Barlett \& Brown, 1999; Brown, et

316 al., 2007; Bragg, et al., 2015). It is possible that decreased tolerance of radial compared to

317 coccygeal measurement resulted in a site-associated white coat effect, although there was no

318 difference in anxiety scores or apparent tolerance of the procedure between the two sites. While 
319 no correlation was observed between anxiety score and SAP measurement or heart rate for either

320 site, previous studies in human medicine have revealed that suppression of anger in both men

321 (Mills \& Dimsdale, 1993) and women (Thomas, 1997) can result in elevated blood pressure. It

322 is possible that dogs that internalize stress, resulting in outwardly calm demeanors, might be

323 more affected by white coat hypertension than overtly anxious dogs. Resting heart rates taken in

324 hospital have been found to be significantly higher than measurements taken in the home

325 environment for both cats (Belew, Bartlett \& Brown, 1999) and dogs (Remillard et al., 1991;

326 Kallet, Cowgill \& Kass, 1997; Bragg, et al., 2015). Although some studies observed concurrent

327 increases in blood pressure and heart rate in a clinic setting (Belew, Barlett \& Brown, 1999;

328 Bragg, et al., 2015), at least one study found no correlation between increases in heart rate and

329 blood pressure (Remillard et al., 1991). Although our study did not compare the heart rates at

330 home with those obtained upon entry into the hospital, heart rates taken before and after

331 acclimation and SAP measurement were compared. Heart rates declined significantly during

332 data collection, and heart rates at the conclusion of SAP measurement at the radial site were

333 significantly lower than heart rates at the end of acclimation or the beginning of data collection.

334 Importantly, there was no significant difference between the first SAP reading taken in each dog

335 at either site $v s$. the mean of the following 5 in spite of continuing changes in heart rate. This

336 suggests that the positive association between average heart rate and mean SAP at each

337 measurement site is more likely due to underlying physiologic processes $v s$. persistent white-coat 338 effects.

339 Given caseload and personnel constraints in many practices, it can be challenging to 340 consistently make time for adequate acclimation, as well as collection of a full series of SAP 341 measurements. The importance of measuring SAP prior to other interventions and allowing 
342 patients to acclimate to their surroundings - including veterinary personnel- prior to blood

343 pressure measurement cannot be overstated, however. In one study comparing blood pressure

344 measurement results for healthy cats and cats with experimentally-induced chronic kidney

345 disease (Belew, Barlett \& Brown, 1999), white-coat associated blood pressure increases were

346 more marked and less quick to normalize in cats with kidney disease. Because routine blood

347 pressure measurement is recommended for animals diagnosed with diseases associated with

348 secondary hypertension (Acierno \& Labato, 2004; Henik, Dolson \& Wenholz, 2005; Brown et

349 al., 2007) and they often are subjected to more frequent veterinary visits for diagnostic sampling

350 and medical procedures, such patients are particularly vulnerable to being misdiagnosed with

351 hypertension if not allowed adequate acclimation and/or if other procedures are performed prior

352 to blood pressure measurement. Although the ACVIM consensus statement (Brown et al., 2007)

353 recommends collecting a total of 3 to 7 measurements and discarding the first before determining

354 the average SAP, there was no clinical or statistical difference between the first measurement,

355 average of BP 2-6, or BP 1-6 in this study. This suggests that a single SAP measurement could

356 be used in dogs with limited concern for reproducibility of the results, assuming that adequate

357 acclimation of the subject occurs. In contrast, failure to perform an adequate acclimation cannot

358 be overcome by collection of additional readings - as demonstrated by the 2 subjects in this

359 study with previously diagnosed, apparently refractory hypertension that were repeatably

360 normotensive, both during and after the study, when acclimated for 10 minutes with technical

361 personnel present. For these reasons, in cases where it is not feasible to do both, acclimation of

362 the patient with personnel should be prioritized over collection of multiple readings in dogs.

363 Current guidelines for indirect measurement of blood pressure in dogs recommend

364 selecting a cuff size that is approximately $40 \%$ of the appendage circumference (Brown et al., 
365 2007). Undersized or oversized blood pressure cuffs have been shown to falsely elevate or lower

366 indirect blood pressure measurements, respectively (Valtonen \& Eriksson, 1970; Bodey et al.,

367 1994; Sparkes et al., 1999). Although appendage circumference was measured in this study to

368 allow selection of the ideal cuff size, actual percent circumference of the cuff ranged from 30-

$36966 \%$ due to lack of availability of half-sizes. In spite of this, no association was found between

370 cuff size as a percentage of circumference and SAP results at either site. This suggests that use of

371 a suboptimal percentage due to cuff size limitations should have minimal clinical impact on

372 blood pressure measurement.

373 One limitation of this study was the lack of direct arterial measurements to compare to

374 indirect SAP measurement results. However, as discussed above, direct SAP measurement is

375 invasive and can be painful, limiting its utility in evaluation of blood pressure in conscious

376 animals on an outpatient basis. Additionally, direct SAP results vary depending on the vessel

377 used and position of the animal, as demonstrated by Acierno et al. (2015). Thus, for comparison

378 to direct SAP results to be valid, direct and indirect measurements would need to be taken at both

379 the radial and coccygeal sites. Because the coccygeal artery is not a paired artery, simultaneous

380 collection of direct arterial and indirect SAP measurements would not be possible. To avoid

381 confounding impacts of arterial catheterization on local vascular tone, direct and indirect SAP

382 measurements would either need to be collected on separate days or the study restructured so all

383 indirect measurements were obtained prior to direct measurements. Although simultaneous

384 measurements would be possible for the radial arteries, comparisons would be confounded by

385 body position (both arteries cannot be 'up' at the same time). Although a crossover design could

386 be employed, 'white coat' induced by arterial catheterization would be anticipated to confound 
387 indirect SAP results taken after direct measurements. For all these reasons, direct SAP 388 measurements were not collected. Instead of using a crossover design, simultaneous measurement at both sites could have been considered to decrease any discordance due to moment by moment variation in blood

391 pressure. This would have required either collection of all measurements with the foot elevated 392 and the dog in a standing position, which is not a commonly used or recommended position for 393 radial SAP measurement, or in lateral recumbency. Because the authors have found the coccygeal site to be particularly useful for accurate SAP measurement in dogs resistant to sitting or lying down, collection of coccygeal measurements in lateral recumbency was considered to 396 limit the clinical applicability of the results. Finally, simultaneous cuff inflation at two sites is not consistent with standard measurement technique in clinical practice and it is unknown how that would have affected measurement-associated white-coat effect. The decision to maximize clinical relevance of the results by using a crossover design instead of collecting pressures simultaneously is unlikely to have substantively influenced the results given the lack of significant differences (statistically or clinically) among measurement results series (first measurement vs 2-6 vs 1-6) for either site and lack of period effects (which would have suggested a change in pressure in the time intervening between the 2 measurement series. There were a number of additional limitations in the present study. In accordance with 405 ACVIM guidelines, headphones were used for collection of SAP measurements in this study. 406 The results, therefore, cannot be directly translated to other techniques including Doppler 407 measurements taken without the use of headphones. A limited number of under-conditioned and 408 sarcopenic dogs were available for enrollment in the study, with only 8 dogs in the BCS 1-2 409 group and only 6 dogs in the MCS 0-1 group. These demographics reflect the local population, 
410 which primarily includes pets of ideal or over-conditioned BCS. Although patients were

411 recruited from both a healthy and an unhealthy population, most animals with higher BCS and

412 MCS were not clinically ill. It is possible that diseases associated with hypertension, muscle

413 wasting, and underconditioning-like chronic kidney disease-might affect accurate blood

414 pressure measurement differently at 1 or both of the measurement sites. Six dogs in the study had

415 hyperadrenocorticism (see data file), but there were few or no dogs enrolled that had other

416 diseases, such as chronic kidney disease and diabetes mellitus, that commonly cause

417 hypertension. Further study of all 3 factors together in a larger population of dogs with such

418 disease processes is, therefore, recommended. Finally, a limited number of critically ill or

419 hypotensive dogs were enrolled in this study, with only 2 dogs being classified as hypotensive.

420 Because such animals often assume a laterally recumbent position, results for coccygeal artery

421 measurements should be extrapolated to that patient population with caution.

422

423 Conclusions

$424 \quad$ Indirect Doppler flow SAP measurements obtained at the radial and coccygeal artery sites

425 were only moderately correlated. Mean radial measurements were $9 \mathrm{mmHg}$ higher than

426 coccygeal measurements, but discordance occurred in both directions. No association was found

427 between measurements obtained at either site and age, weight, BCS, MCS, and anxiety score.

428 Interestingly, no association was found between cuff size and blood pressure measurement at

429 either site, suggesting that use of a cuff outside the recommended range might have little impact

430 on the accuracy of results in a clinical setting.

431 Heart rate decreased throughout each data collection series, with results at the conclusion

432 of SAP measurement significantly lower than those obtained at the beginning of SAP 
433 determination. However, there was no significant difference between the first SAP measurement,

434 the mean of the additional 5 readings (BP 2-6), and the mean of all 6 readings (BP 1-6). In

435 clinical situations where time is limited, the authors recommend that fewer blood pressure

436 measurements be obtained instead of decreasing time devoted to patient acclimation to the

437 environment and personnel.

438

439 Footnotes

440 a. https://www.random.org. Accessed May 15, 2016.

441 b. Riester Ri-san ${ }^{\circledR}$ aneroid sphygmomanometer, Riestar Direct, Ventura, California, United

442 States.

443 c. Model 811-B Doppler ultrasonic Flow Detector with flat infant probe, Parks Medical

444 Electronics, Inc., Aloha, Oregon, United States.

445 d. MedCalc 15.8 MedCalc Software, Ostend, Belgium.

446 e. SAS 9.4 release TS1M3, SAS Institute Inc., Cary, North Carolina, United States.

447 f. IBM SPSS Statistics for Windows, version 24, IBM Corp., Armonk, NY, United States.

448

449 References

450

451 Acierno MJ, Labato MA. 2004. Hypertension in dogs and cats. Compendium on Continuing

452 Education for the Practicing Veterinarian 26(5):336-345.

453 
454 Acierno MJ, Domingues ME, Ramos SJ, Shelby AM, da Cunha AF. 2015. Comparison of

455 directly measured arterial blood pressure at various anatomic locations in anesthetized dogs.

456 American Journal of Veterinary Research 76(3):266-271. DOI: 10.2460/ajvr.76.3.266.

457

458 Baldwin K, Bartges J, Buffington T, Freeman LM, Grabow M, Legred J, Ostwald Jr D. 2010.

459 AAHA nutritional assessment guidelines for dogs and cats. Journal of the American Animal

460 Hospital Association 46(4): 285-296. DOI: 10.5326/0460285.

461

462 Belew AM, Barlett T, Brown SA. 1999. Evaluation of the white-coat effect in cats. Journal of

463 Veterinary Internal Medicine 13(2):134-142. DOI: 10.1111/j.1939-1676.1999.tb01141.x.

464

465 Bodey AR, Young LE, Bartram DH, Diamond MJ, Michell AR. 1994. A comparison of direct

466 and indirect (oscillometric) measurements of arterial blood pressure in anesthetized dogs, using

467 tail and limb cuffs. Research in Veterinary Science 57(3):265-269. DOI: 10.1016/0034-

$468 \quad 5288(94) 90116-3$.

469

470 Bodey AR, Michell AR, Bovee KC, Buranakurl C, Garg T. 1996. Comparison of direct and

471 indirect (oscillometric) measurements of arterial blood pressure in conscious dogs. Research in

472 Veterinary Science 61(1):17-21. DOI: 10.1016/S0034-5288(96)90104-6.

473

474 Bodey AR, Michell AR. 1996. Epidemiological study of blood pressure in domestic dogs.

475 Journal of Small Animal Practice 37(3):116-125. DOI: 10.1111/j.1748-5827.1996.tb02358.x

476 
477 Bosiack AP, Mann FA, Dodam JR, Wagner-Mann CC, Branson KR. 2010. Comparison of

478 ultrasonic Doppler flow monitor, oscillometric, and direct arterial blood pressure measurements

479 in ill dogs. Journal of Veterinary Emergency and Critical Care 20(2):207-215. DOI:

$480 \quad 10.1111 / \mathrm{j} .1476-4431.2010 .00520 . \mathrm{x}$

481

482 Bragg RF, Bennett JS, Cummings A, Quimby JM. 2015. Evaluation of the effects of hospital 483 visit stress on physiologic variables in dogs. Journal of the American Veterinary Medical 484 Association 246(2):212-215. DOI: 10.2460/javma.246.2.212.

485

486 Brown S, Atkins C, Bagley R, Carr A, Cowgill L, Davidson M, Egner B, Elliot J, Henik R, 487 Labato M, Littman M, Polzin D, Ross L, Snyder P, Stepien R. 2007. Guidelines for the 488 identification, evaluation, and management or systemic hypertension in dogs and cats. Journal of 489 Veterinary Internal Medicine 21:542-558.

490

491 Carr AP, Egner B. 2009. Blood pressure in small animals - Part 2: Hypertension - target organ 492 damage, heart and kidney. European Journal of Companion Animal Practice 19(1):1-5. 493

494 Gains MJ, Grodecki KM, Jacobs RM, Dyson D, Foster RA. 1995. Comparison of direct and 495 indirect blood pressure measurements in anesthetized dogs. Canadian Journal of Veterinary 496 Research 59(3):238-240.

497

498 Henik RA, Dolson MK, Wenholz LJ. 2005. How to obtain a blood pressure measurement.

499 Clinical Techniques in Small Animal Practice 20(3):144-150. DOI: 10.1053/j.ctsap.2005.05.005. 
500

501 Hsiang TY, Lien YH, Huang HP. 2008. Indirect measurement of systemic blood pressure in

502 conscious dogs in a clinical setting. Journal of Veterinary Medical Science 70(5):449-453. DOI:

$503 \quad 10.1292 / j v m s .70 .449$.

504

505 Kallet AJ, Cowgill LD, Kass PH. 1997. Comparison of blood pressure measurements obtained in 506 dogs by use of indirect oscillometry in a veterinary clinic versus at home. Journal of the 507 American Veterinary Medical Association 210(5):651-654.

508

509 Kenney EM, Rozanski EA, Rush JE, deLaforcade-Buress AM, Berg JR, Silverstein DC, 510 Montealegre CD, Jutkowitz LA, Adamantos S, Ovbey DH, Boysen SR, Shaw SP. 2010.

511 Association between outcome and organ system dysfunction in dogs with sepsis: 114 cases 512 (2003-2007). Journal of the American Veterinary Medical Association 236(1):83-87. DOI:

$513 \quad 10.2460 /$ javma.236.1.83.

514

515 King LG, Wohl JS, Manning AM, Hackner SG, Raffe RM, Maislin G. 2001. Evaluation of the 516 survival prediction index as a model of risk stratification for clinical research in dogs admitted to 517 intensive care units at four locations. American Journal of Veterinary Research 62(6):948-954. 518 DOI: $10.2460 / a j v r .2001 .62 .948$.

519

520 Michel KE, Anderson W, Cupp C, Laflamme DP. 2011. Correlation of a feline muscle mass

521 score with body composition determined by dual-energy x-ray absorptiometry. British Journal of 522 Veterinary Nutrition 106(S1):S57-S59. DOI: 10.1017/S000711451100050X. 
524 Mills PJ, Dimsdale JE. 1993. Anger suppression: its relationship to beta-adrenergic receptor

525 sensitivity and stress-induced changes in blood pressure. Psychological Medicine 23(3):673-678.

526

527 Remillard RL, Ross JN, Eddy JB. 1991. Variance of indirect blood pressure measurements and 528 prevalence of hypertension in clinically normal dogs. American Journal of Veterinary Research 529 52(4):561-565.

530

531 Rondeau DA, Mackalonis ME, Hess RS. 2013. Effect of body position on indirect measurement 532 of systolic arterial blood pressure in dogs. Journal of the American Veterinary Medical 533 Association 242(11):1523-1527. DOI: 10.2460/javma.242.11.1523.

534

535 Sawyer DC, Guikema AH, Siegel EM. 2004. Evaluation of a new oscillometric blood pressure 536 monitor in isofluorane-anesthetized dogs. Veterinary Anaesthesia and Analgesia 31(1):27-39. 537 DOI: 10.1111/j.1467-2995.2004.00141.x.

538

539 Scansen BA, Vitt J, Chew DJ, Schober KE, Bonagura JD. 2014. Comparison of forelimb and 540 hindlimb systolic blood pressures and proteinuria in healthy Shetland sheepdogs. Journal of 541 Veterinary Internal Medicine 28(2):277-283. DOI: 10.111/jvim/12289.

542

543 Silverstein DC, Wininger FA, Shofer FS, King LG. 2008. Relationship between Doppler blood 544 pressure and survival or critical response to treatment in critically ill cats: 83 cases (2003-2004). 
545 Journal of the American Veterinary Medical Association 232(6):893-897. DOI:

546 10.2460/javma.232.6.893.

547

548 Silverstein DC, Kleiner J, Drobatz KJ. 2012. Effectiveness of IV fluid resuscitation in the

549 emergency room for treatment of hypotension in dogs: 35 cases (2000-2010). Journal of

550 Veterinary Emergency and Critical Care 22(6):666-673. DOI: 10.1111/j.1476-

$551 \quad 4431.2012 .00822 . x$.

552

553 Simpson KE, McCann TM, Bommer NX, Pereira YM, Corston C, Reed N, Gunn-Moore DA.

554 2007. Retrospective analysis of selected predictors of mortality within a veterinary intensive care 555 unit. Journal of Feline Medicine and Surgery 9(5): 364-368. DOI: 10.1016/j.jfms.2007.03.001.

556

557 Sparkes AH, Caney SMA, King MCA, Gruffydd-Jones TJ. 1999. Inter- and intraindividual

558 variation in Doppler ultrasonic indirect blood pressure measurements in healthy cats. Journal of

559 Veterinary Internal Medicine 13(4):314-318. DOI: 10.1111/j.1939-1676.1999.tb02187.x.

560

561 Thomas SP. 1997. Women's anger: relationship of suppression to blood pressure. Nursing

562 Research 46(6):324-330.

563

564 Vachon C, Belanger MC, Burns PM. 2014. Evaluation of oscillometric and Doppler ultrasonic

565 devices for blood pressure measurements in anesthetized and conscious dogs. Research in

566 Veterinary Science 97(1):111-117. DOI: 10.1016/j.rvsc.2014.05.003.

567 
568 Valtonen MH, Eriksson, LM. 1970. The effect of cuff width on accuracy of indirect

569 measurement of blood pressure in dogs. Research in Veterinary Science 11:358-362.

570

571 Wernick MB, Hopfner RM, Francey T, Howard J. 2012. Comparison of arterial blood pressure

572 measurements and hypertension scores obtained by use of three indirect measurement devices in

573 hospitalized dogs. Journal of the American Veterinary Medical Association 240(8):962-968.

574 DOI: 10.2460/javma.240.8.962. 


\section{Table $\mathbf{1}$ (on next page)}

Baseline demographics for 62 privately-owned conscious dogs in which indirect radial and coccygeal systolic arterial blood pressure (SAP) measurements were collected.

Values are reported as median (range). BCS = body condition score. MCS $=$ muscle condition

score. *The mean of scores for 2 investigators (JCW, DIM) were used for statistical analyses. 
1

2

\begin{tabular}{|c|c|c|c|c|c|c|}
\hline \multirow[b]{2}{*}{ Variable } & \multicolumn{5}{|c|}{ Enrollment BCS } & \multirow[b]{2}{*}{$\begin{array}{c}\text { All dogs (n } \\
=62)\end{array}$} \\
\hline & $1(n=2)$ & $2(n=6)$ & $3(n=20)$ & $4(n=20$ & $5(n=14)$ & \\
\hline $\begin{array}{l}\text { Age } \\
\text { (years) }\end{array}$ & $\begin{array}{c}13.5(12- \\
15)\end{array}$ & $10(3-11)$ & $7(1-13)$ & $7(3-14)$ & $9.5(5-14)$ & $8(1-15)$ \\
\hline Gender & $2 \mathrm{MC}$ & $1 \mathrm{FS}, 5 \mathrm{MC}$ & $\begin{array}{c}10 \mathrm{FS}, 11 \\
\mathrm{MC}\end{array}$ & $\begin{array}{c}8 \mathrm{FS}, 11 \\
\mathrm{MC}\end{array}$ & $8 \mathrm{FS}, 6 \mathrm{MC}$ & $\begin{array}{c}27 \mathrm{FS}, 35 \\
\mathrm{MC}\end{array}$ \\
\hline $\begin{array}{l}\text { Weight } \\
(\mathrm{kg})\end{array}$ & $\begin{array}{c}22.7(13.3- \\
31)\end{array}$ & $\begin{array}{c}7.1(2.7- \\
41.4)\end{array}$ & $\begin{array}{c}20.9(2.7- \\
54.3)\end{array}$ & $\begin{array}{l}16.2(2.3- \\
50.4)\end{array}$ & $\begin{array}{c}14.1(4.9- \\
61.3)\end{array}$ & $\begin{array}{c}18.6(2.1- \\
61.3)\end{array}$ \\
\hline $\begin{array}{l}\text { Anxiety } \\
\text { score }\end{array}$ & 1 (NA) & $1(1-3)$ & $1(0-2)$ & $1(0-2)$ & $1(0-3)$ & $1(0-3)$ \\
\hline $\begin{array}{l}\text { Mean } \\
\text { BCS* }\end{array}$ & $\begin{array}{c}1.25(1- \\
1.5)\end{array}$ & $2(1.5-2)$ & $3(2-3.5)$ & $4(3-5)$ & $5(4.5-5)$ & $\begin{array}{c}3.5(1.0- \\
5.0)\end{array}$ \\
\hline $\begin{array}{l}\text { Mean } \\
\text { MCS* }\end{array}$ & $\begin{array}{c}0.25(0- \\
0.5)\end{array}$ & $2(1-3)$ & $3(1-3)$ & $3(1-3)$ & $2.5(1.5-3)$ & $\begin{array}{c}3.0(0.0- \\
3.0)\end{array}$ \\
\hline $\begin{array}{l}\text { Radial } \\
\text { SAP } \\
(\mathrm{mmHg})\end{array}$ & $120 \pm 5.2$ & $139 \pm 18.8$ & $126 \pm 20.5$ & $126 \pm 16.4$ & $136 \pm 15.4$ & $130 \pm 18.0$ \\
\hline $\begin{array}{l}\text { Coccygeal } \\
\text { SAP } \\
(\mathrm{mmHg})\end{array}$ & $110 \pm 29.6$ & $129 \pm 17.5$ & $117 \pm 17.5$ & $116 \pm 19.0$ & $129 \pm 21.2$ & $120 \pm 18.8$ \\
\hline
\end{tabular}

3

4 
Figure 1

Correlation between mean indirect radial and coccygeal systolic arterial blood pressure measurements collected via Doppler ultrasonic flow detector for 62 privately-owned conscious dogs.

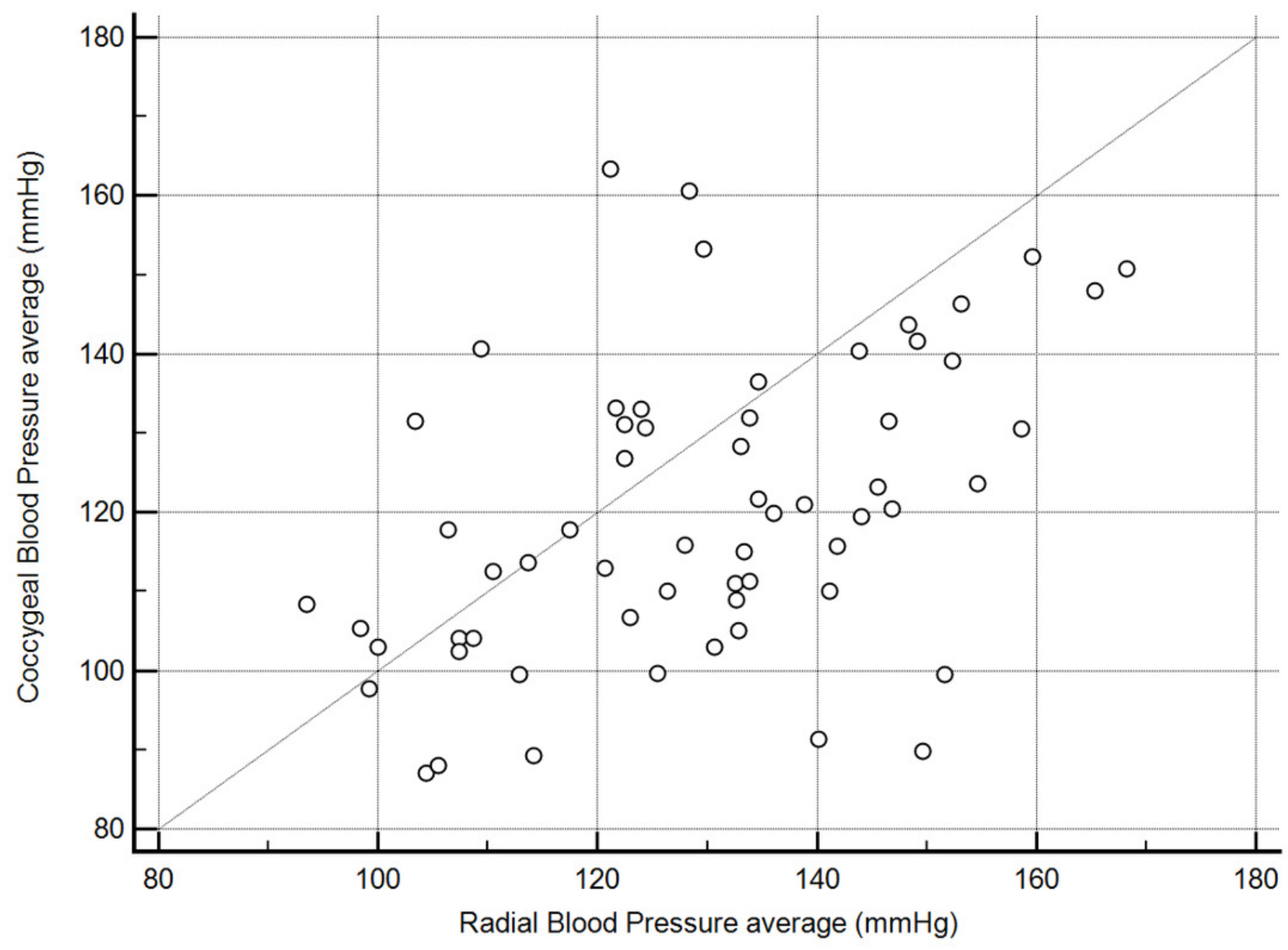


Figure 2

Mean indirect radial and coccygeal systolic arterial blood pressure measurements collected via Doppler ultrasonic flow detector in 62 privately-owned conscious dogs.

Dogs are grouped by enrollment body condition score (BCS) assignment. The open circles represent measurements taken using the coccygeal artery, whereas closed triangles represent measurements taken using the radial artery.

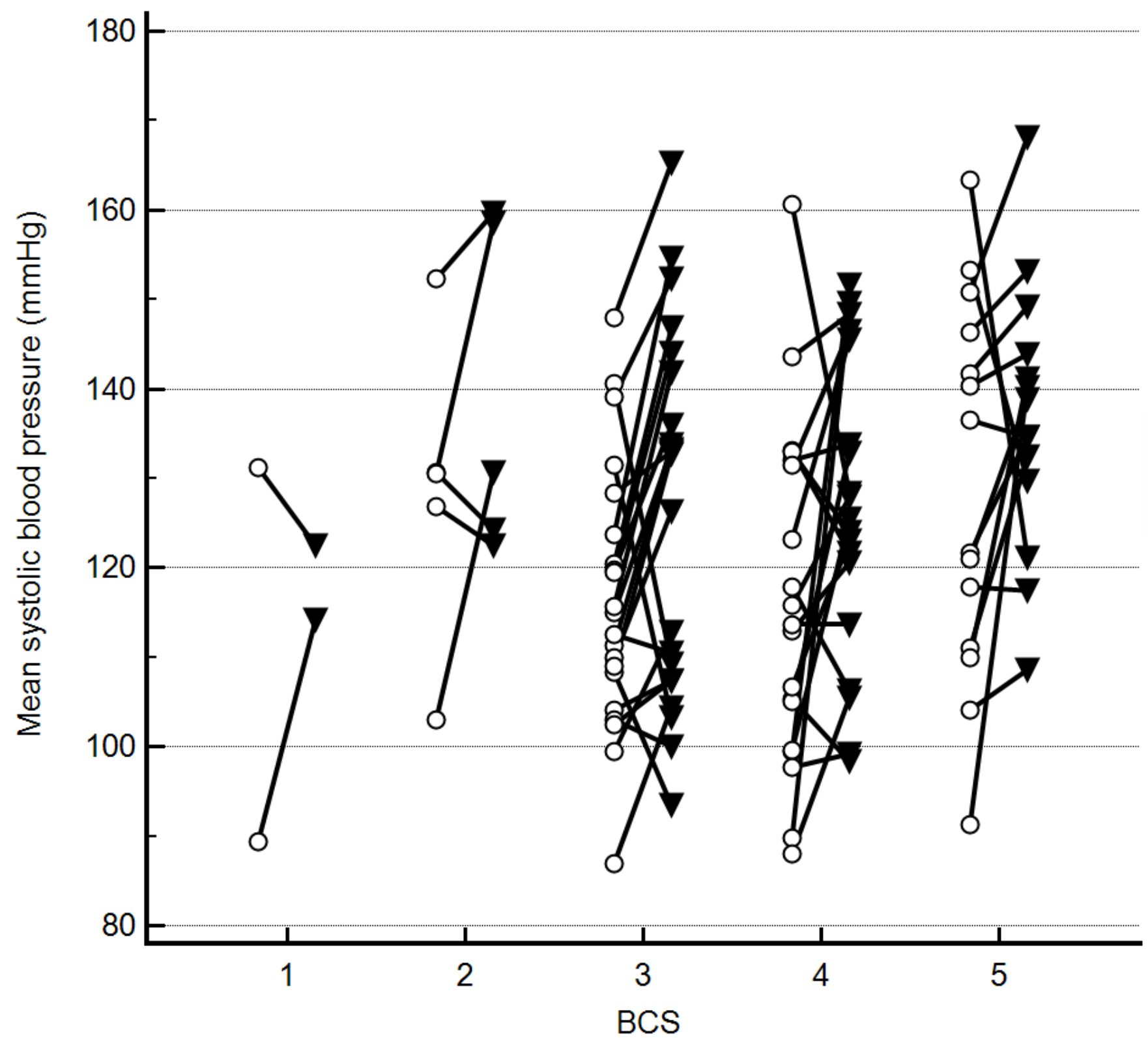

